Cultures \& Conflits

64 | hiver 2006

Identifier et surveiller

\title{
Créer une Révolution dans les affaires militaires : mode d'emploi
}

\section{Christophe Wasinski}

\section{(2) OpenEdition \\ 1 Journals}

\section{Édition électronique}

URL : http://journals.openedition.org/conflits/2135

DOI : $10.4000 /$ conflits.2135

ISSN : $1777-5345$

Éditeur :

CCLS - Centre d'études sur les conflits lilberté et sécurité, L'Harmattan

\section{Édition imprimée}

Date de publication : 20 décembre 2006

Pagination : 149-164

ISBN : 978-2-296-02667-4

ISSN : 1157-996X

\section{Référence électronique}

Christophe Wasinski, «Créer une Révolution dans les affaires militaires : mode d'emploi », Cultures \& Conflits [En ligne], 64 | hiver 2006, mis en ligne le 06 mars 2007, consulté le 30 mars 2021. URL : http:// journals.openedition.org/conflits/2135; DOl : https://doi.org/10.4000/conflits.2135

Ce document a été généré automatiquement le 30 mars 2021.

Creative Commons License 


\title{
Créer une Révolution dans les affaires militaires : mode d'emploi
}

\author{
Christophe Wasinski
}

1 Comment créer une Revolution in Military Affairs ${ }^{1}$, c'est-à-dire comment construire un projet technique qui va donner sens et intégrer en un tout cohérent des outils militaires (armements ou systèmes d'armes) ? C'est la question que soulève cet article. En effet, loin d'être évidente, la notion manque toujours d'une définition officielle ${ }^{2}$ et, par conséquent, d'une réelle stabilité ${ }^{3}$.

2 Dès lors, il paraît intéressant de jeter un autre regard investigateur sur la RMA, qui soit inspiré par celui que Bruno Latour a posé sur le projet "Aramis ${ }^{4}$ ». Dans son étude, Latour a tenté, par une analyse sociologique extrêmement fine, de déconstruire un projet technique. Le cas envisagé facilitait une telle approche, menée sur le mode anthropologique, réalisée entre autres à partir d'entretiens et de documents de première main. Mais "Aramis" ne baignait pas dans une atmosphère de secret. A contrario, la RMA est plus rétive à une telle approche. Comme nombre de projets militaires, elle peut comporter des zones d'ombre, que l'on justifiera éventuellement à tort ou à raison - par des motifs de sécurité. D’autre part, ce travail exploratoire n'a pas les prétentions de l'ouvrage de Latour. Il se veut avant tout un contrepoint aux discours contemporains sur la RMA et désire poser la question de sa fabrication sur un mode discursif et bureaucratique ${ }^{5}$.

Un homme, un mythe, une institution

3 Le concept de RMA trouve son origine dans trois éléments. Il y a d'abord un homme, Andrew (Andy) W. Marshall qui «inventa » le concept en $1993^{6}$. Il y a ensuite une institution : l'Office of Net Assessment (ONA), dont Marshall est le responsable. Il y a enfin un mythe, qui rassemble les deux premiers éléments. En effet, il est difficile de ne pas raconter l'histoire de la RMA sans prendre conscience des dimensions mythiques qui l'entourent. Cette narration a été mise en évidence par quelques journalistes américains qui ont eu l'occasion de croiser Andy Marshall, le patron de l'ONA.

4 C'est en 1973 que le mystérieux Andy Marshall est nommé à la tête de l'ONA, nouvellement créé. Les informations relatives à la carrière de l'homme restent 
fragmentaires. Marshall a grandi à Detroit et a obtenu un diplôme d'économie de l'université de Chicago. Il obtient un poste en 1949 à la RAND Corporation. Un des plus importants think tanks contractant avec le Pentagone lors de la Guerre froide, la RAND fut la principale matrice de production stratégique en rapport avec les armements nucléaires ${ }^{7}$. Marshall y travaille pendant près de vingt-trois ans, mais laisse très peu de traces écrites de son passage. Ces études seraient peut-être encore classifiées. En 1972, il est appelé par Kissinger pour travailler au sein du National Security Council, mais n'y reste pas longtemps. Son ami James Schlesinger, alors secrétaire à la Défense, également un ancien de la RAND, le fait enfin nommer par Nixon à la direction de l'ONA. Ce centre de réflexion interne au Pentagone a pour vocation de penser aux moyens de faire face aux futures menaces qui pourraient peser contre des Etats-Unis. Marshall parvient à rester au sein du Pentagone sous les présidences Ford, Carter, Reagan, Bush, Clinton et sous l'actuelle administration Bush. Il s'intéresse apparemment à la question de la défense antimissile pendant les années 1970, évalue la puissance soviétique, défend le maintien des budgets militaires, etc. Tout ceci reste de l'ordre de l'hypothèse, car là encore, il est extrêmement difficile de retrouver des traces écrites du travail de Marshall. Notons qu'il participe aussi, beaucoup plus récemment, à la rédaction du rapport Rumsfeld en faveur de l'élaboration d'une National Missile Defense ${ }^{8}$.

5 Au final, Marshall dispose d'un curriculum à la fois étoffé et nébuleux. A côté de cela, il est révélateur de voir comment l'homme est présenté publiquement. Aujourd'hui âgé de plus de 80 ans, Marshall est surnommé « Yoda » en référence au sage personnage de Star Wars. On évoque l'existence d'une Church of St. Andrew à son nom au sein du ministère de la Défense ${ }^{9}$. L'étonnante image de longévité et de solidité de sa carrière se trouve renforcée par la tentative avortée du secrétaire à la Défense William S. Cohen, en 1997, sous l'administration Clinton, de placer l'ONA sur une «voie de garage». Il était alors question de transférer l'institution du Pentagone vers la National Defense University, c'est-à-dire en périphérie du pouvoir. Une levée de boucliers s'ensuivit et les prises de position des amis et disciples de Marshall ont eu tôt fait de dissuader Cohen.

6 Par ailleurs, le mythe se nourrit de l'aspect prophétique des " paroles » de l'homme ${ }^{10}$. « Paroles » entre guillemets car Marshall a la réputation d'être effacé, voire timide. Ses interventions et ses citations paraissent comme autant de moments exceptionnels. L'aspect prophétique, lui, est par exemple mis en évidence au travers d'une anecdote, à valeur contestée. Marshall aurait fait preuve d'une grande préséance au cours des années 1980 en se rendant compte que le SIDA pouvait devenir un problème de sécurité nationale. Il aurait contacté le CDC (Centers for Disease Control) pour que celui-ci prenne le problème au sérieux. Mais, contacté par le journaliste d'investigation Jason Vest, le CDC n'a nullement confirmé cette histoire ${ }^{11}$.

7 L'aspect prophétique de sa réflexion se retrouve également dans sa vision géopolitique. Marshall anticipe une confrontation entre les Etats-Unis et la Chine et pense que, d'ici 2025, l'Inde aura plus d'importance que la Russie dans le jeu des relations internationales. Pour faire face à ces évolutions, il se montre favorable à une force aérienne davantage centrée sur les missiles et la défense antimissile. Selon lui, la Navy devrait être composée de sous-marins et de navires de surface permettant de convoyer et d'assister les troupes qui doivent débarquer - un rôle qui subordonne la Navy aux composantes terrestres. L'armée de terre, elle, devrait être divisée en deux : une force 
de grande ampleur pour les circonstances non prévues (" contingencies») et une force techniquement moins développée pour les opérations de maintien de la paix.

Ces éléments nous amènent à une première série de remarques. Dans la plupart des narrations de types historique, sociologique ou journalistique, il est de mise de poser un contexte. Le problème est que tout contexte est lui-même le fait d'une construction intellectuelle - à laquelle les éléments qui précèdent n'échappent pas. Le contexte laisse supposer l'existence de déterminants reconstruits a posteriori, qui peuvent être de nature matérielle, technique, événementielle mais aussi liés aux acteurs. Tout comme la technique, les acteurs sont le fruit d'élaborations humaines. Idéalement, comme le souligne Latour, il faut laisser les acteurs se dévoiler d'eux-mêmes et non postuler leur existence a priori. Il y a acteur à partir du moment où une conscience d'acteur apparaît. Plus encore, la conscience de l'acteur ne provient pas uniquement de données de type biographique ou socio-historique engrangées dans l'esprit de ce dernier. La construction de l'acteur est aussi le résultat d'interactions avec d'autres acteurs et avec l'environnement ${ }^{12}$.

9 Dans le cas présent, nous nous trouvons limités car nous n'avons pas directement consulté Andy Marshall. Par ailleurs, il est assez difficile de savoir jusqu'à quel point l'homme utilise son image mythique et jusqu'à quel point il se concevait, par avance, comme un acteur doté d'une stratégie dans le débat à venir sur la RMA. Mais cela ne signifie pas que rien n'a été appris. Plus précisément, c'est bien la construction d'une origine à la RMA qui a été mise en exergue. L'énoncé originel pourra être situé dans le temps et dans l'espace grâce à ces informations ${ }^{13}$. A partir de là, il sera possible de montrer les premiers pas du concept. De plus, cela a permis d'en savoir davantage sur la manière dont l'entourage d'Andy Marshall le perçoit. Les récits journalistiques évoquant son rôle sont le reflet des échos qui circulent à son propos. Ils sont l'émanation de la satisfaction ou du mécontentement de tel officier ou de tel « intellectuel de la défense » à son encontre. Les informations biographiques ne sont pas évoquées pour leur seule valeur historique mais afin d'interpréter le présent, ce qui signifie qu'elles ont un impact tant sur la question de la fabrication du contexte que sur celle de la fabrication des acteurs. Ce contexte montre lui-même qu'il y a potentiellement désaccord. Marshall est tour à tour décrit comme un génie ou un faux prophète, un conseiller du prince œuvrant dans l'ombre à la réalisation de sombres desseins ou comme celui qui dotera l'Amérique d'une plus grande sécurité. Le mythe est probablement moins le résultat de Marshall lui-même que de son entourage. N'oublions pourtant pas que l'une des fonctions essentielles du mythe est de produire une narration qui va occulter l'importance des rapports de force ${ }^{14}$. Or, la question de la RMA se trouve, justement, directement liée à des rapports de force.

Les premiers pas d'un concept

10 C'est en 1993 qu'Andy Marshall utilise pour la première fois l'expression « Révolution dans les affaires militaires» dans un document officiel. Il aurait été inspiré par les travaux du maréchal soviétique Nicolaï Ogarkov. Ce dernier a introduit la notion de « révolution technique militaire » au début des années 1980. Selon lui, l'introduction de systèmes militaires de reconnaissance et d'attaques automatisés, de moyens de contrôle électroniques et de munitions longue-portée donnait un grand avantage aux forces conventionnelles, dont le potentiel destructeur commençait à s'approcher des armes nucléaires. La narration «populaire» indique que Marshall reprend le concept de révolution technique et l'élargit pour la transformer en RMA en 1993, après la fin de 
la guerre du Golfe. Cette dernière avait été l'occasion pour les forces armées américaines de faire étalage de leurs moyens techniques.

11 Certaines sources indiquent que Marshall pensait déjà au concept de la RMA en 1991. Néanmoins, le concept n'est formalisé qu'en 1993 avec la distribution d'un court mémorandum (intitulé Some Thoughts on Military Revolution, juillet 1993) signé de la main de Marshall, avec mention à l'ONA, au sein du Pentagone. Le secrétaire à la Défense de l'époque, William Perry, approuve la note en septembre 1993 et, en janvier 1994, fait établir un groupe de réflexion sur le sujet. La première phase consiste à rassembler des informations à propos de l'environnement de défense le plus probable pour les années 2010-2015, l'identification des techniques et des concepts opérationnels les plus prometteurs. Dans un second temps, des war games sont conduits pour évaluer l'impact des découvertes. L'ensemble mène à la réalisation d'un rapport. Peu de temps après ces discussions, le concept se retrouve plus largement diffusé en dehors des murs du Pentagone par une déclaration sur le sujet au sein du Senate Armed Forces Services Committee, en mai 1995.

Ceci étant, il est possible de trouver une définition de la RMA attribuée à Marshall :

«Une Révolution dans les affaires militaires est un changement majeur dans la nature de la guerre [warfare] suite à l'application de nouvelles technologies innovantes qui, combinées à des changements radicaux dans les doctrines militaires et opérationnelles, ainsi que dans les concepts organisationnels, altère fondamentalement le caractère et la conduite des opérations militaires ${ }^{15} \%$.

Plusieurs remarques supplémentaires s'imposent à ce stade. Tout d'abord, un élément quelque peu paradoxal: le concept de RMA trouve son inspiration chez l'adversaire soviétique (Ogarkov). Cela soulève la question du mimétisme stratégique. Plusieurs auteurs - politologues et historiens - ont déjà mis en évidence ce phénomène. Selon certains, les réseaux de socialisation militaire (contacts personnels, lectures, présence lors de manœuvres ou participation à des conférences) jouent un rôle de premier plan dans ce processus. Pour d'autres, la structure internationale impose des contraintes aux acteurs internationaux et tend à égaliser leur comportement, y compris au niveau militaire ${ }^{16}$. Le facteur de mimétisme prend une tournure encore plus étrange si l'on considère que la réaction d'Ogarkov était elle-même inspirée par l'évaluation des capacités américaines (et l'emploi de plus en plus systématique de moyens électroniques depuis la guerre du Vietnam). En d'autres termes, Marshall aurait pris conscience des changements dans l'art de la guerre au travers de la vision soviétique des forces armées américaines.

Un discours tentaculaire

Jusqu'à présent, le nom de Marshall a paru recouvrir l'ensemble des activités de l'ONA. En réalité, ce centre de réflexion est plus que cela. Décrit comme une série de bureaux de taille modeste au sein du Pentagone et composé d'un personnel limité (une douzaine d'individus), l'ONA se présente, après rapide analyse, comme une sorte de courroie d'activation de réseaux intellectuels ${ }^{17}$, qui impliquent à la fois des personnalités politiques, militaires, universitaires et de l'industrie de la Défense.

D'emblée, Marshall est présenté comme un conservateur. On le situe comme un proche du secrétaire de la Défense actuel, Donald Rumsfeld, et de son assistant direct, Paul Wolfowitz. En creusant l'histoire politico-stratégique des Etats-Unis, on remarquera que les liens entre ces personnages tirent une partie de leurs origines au cours de la Guerre froide. L'une des matrices de ces réseaux est originaire de la RAND Corporation, 
organisation issue d'un centre de recherches de la compagnie aéronautique Douglas qui gagne son indépendance en 1949. On fait appel à elle pour réaliser des études, principalement pour le compte de l'US Air Force, dont les plus notables portent sur la stratégie nucléaire. La RAND est un vivier d'intellectuels, surtout rompus à des techniques d'analyses mathématiques (théorie des jeux, recherche opérationnelle, analyse des systèmes) ${ }^{18}$. Parmi les chercheurs en question, l'un mérite une attention particulière, Robert Wohlstetter (1915-1997) ${ }^{19}$. Conservateur pur et dur, il est considéré comme l'un des plus brillants analystes issus de la RAND. Son ambition le conduit à lier des contacts avec le monde politique et à ne pas se cantonner à des questions stratégiques techniques. Il restera cependant dans l'ombre des décisions importantes en matière de politique de défense des Etats-Unis ${ }^{20}$.

16 Mais, si le nom de Wohlstetter mérite tout particulièrement d'être mentionné aujourd'hui, c'est parce qu'il représente en quelque sorte un point de repère généalogique à partir duquel le mouvement conservateur d'abord, et néoconservateur ensuite, de la politique de sécurité américaine a su prendre appui ${ }^{21}$. Wohlstetter est ainsi présenté comme le mentor de Paul Wolfowitz, l'actuel bras droit de Rumsfeld, et de Richard Perle (dont il fut le beau-père) et l'un des rédacteurs de l'actuel document National Security Strategy - qui insiste sur la notion de guerre préventive. Ajoutons que Wohlstetter a également entretenu des contacts avec Donald Rumsfeld à partir des années 1970. Bien entendu, de par leur passage commun à la RAND, Wohlstetter a connu Andy Marshall.

17 Le réseau de l'ONA connaît ensuite une extension auprès de certains chercheurs, civils et militaires, anciennement actifs dans l'ONA, et ayant contribué à diffuser des analyses en faveur de la thèse de la Révolution dans les affaires militaires. On retrouvera la trace de ceux-ci dans le panel d'analystes ayant participé à la rédaction du rapport sur l'emploi des forces aériennes durant la guerre du Golfe de $1991{ }^{22}$. Outre Marshall, on trouve dans ce document le nom du célèbre commentateur stratégique Eliot A. Cohen, actuellement professeur à la John Hopkins University, dans la Paul H. Nitze School of Advanced International Studies (SAIS). Cohen est en effet un ancien de l'ONA, tout comme deux autres membres de la SAIS : Andrew Krepinevich (voir infra) et Thomas Mahnken ${ }^{23}$.

18 On retrouve également Henry S. Rowen, un ancien directeur de la RAND Corporation. Enfin, on constate la présence de Barry D. Watts, ex-pilote de chasse qui a ensuite été professeur de logique philosophique et de mathématiques au sein de l'US Air Force Academy. Il a également travaillé pour le compte de l'Office of Net Assessment avant de se retirer des forces armées en 1986 pour être engagé par le centre d'analyses de la compagnie aéronautique Grumman ${ }^{24}$. Lors de son passage chez Grumman, il continue à pourvoir l'ONA en évaluations. En 2001, il prend les fonctions de Director of Program Analysis and Evaluation. Cet organisme réfléchit aux relations entre achats, budgets, menaces et contributions des Alliés. Ce bureau est censé conseiller directement le secrétaire de la Défense Rumsfeld ${ }^{25}$. Ajoutons que le centre d'analyses de Grumman avait aussi pour membre James (Jim) G. Roche ${ }^{26}$, un autre proche de Marshall, qui a travaillé pour l'ONA de 1975 à 1979. James Roche a depuis lors été nommé Secretary of the Air Force par le président Bush ${ }^{27}$.

19 Le nom de Watts doit encore être mentionné dans la publication d'une série d'ouvrages relatifs aux innovations militaires. Ceux-ci sont dirigés par les historiens militaires Williamson Murray et Allan R. Millet, avec le soutien actif de l'ONA. Les trois ouvrages 
sont intitulés Military Effectiveness (1988), Calculation (1992) et Military Innovation (1996). Ce dernier livre est particulièrement intéressant pour son sous-titre complet: «Military Innovation in the Interwar Period». Il s'agit de mettre en évidence les innovations militaires des années 1920 et 1930 tels que les porte-avions, la préparation aux opérations amphibies, la mécanisation des forces armées terrestres, etc. ${ }^{28}$.

Ajoutons que la revue Joint Forces Quarterly semble aussi se faire le porte-parole des tenants de la RMA. Andrew Marshall y conclut par exemple le RMA Essay Contest, un concours de textes proposé aux membres des forces armées ${ }^{29}$. L'historien militaire Williamson Murray, lui, y présente les résultats d'une conférence tenue à ce propos à l'académie navale de Quantico en avril 1996, conférence ayant rassemblé des célébrités en matière d'histoire militaire: Geoffrey Parker, John Lynn, Dennis Showalter, Holger Herwig, Allan R. Millet, etc. ${ }^{30}$. L'auteur avait rédigé un an plus tôt un autre texte sur le sujet. Dans les notes, on retrouvait des remerciements à Barry Watts ${ }^{31}$.

21 Il faut encore prendre en ligne de compte un autre "intellectuel de la défense »: Stephen Rosen. Professeur à l'université d'Harvard, celui-ci a été l'assistant civil du directeur de l'ONA ainsi que le directeur des affaires politico-militaires du National Security Council sous l'administration Reagan. Il a assuré des missions d'enseignement au Naval War College. Il a lui aussi participé à l'élaboration du rapport Discriminate Deterrence - qui envisageait le futur stratégique des Etats-Unis à la fin de la Guerre froide - et au Gulf War Air Power Survey. Lorsqu'on évoquera la possibilité de transférer Marshall du Pentagone à la National Defense University, Stephen Peter Rosen sera l'un des intellectuels à prendre la défense du patron de l'ONA. Accessoirement, on constatera que la prise de position de Rosen se trouve postée sur le site Internet $d u$ groupe néoconservateur du Project for a New American Century (PNAC) ${ }^{32}$. On retrouve, gravitant autour de ce mouvement, Donald Rumsfeld, Paul Wolfowitz, Jeb Bush, Dick Cheney, Richard Perle ${ }^{33}$. Plus intéressant encore, Rosen est l'auteur d'une thèse, publiée, sur la question de l'innovation militaire ${ }^{34}$. L'une des idées centrales développée est qu'il existe souvent, parmi les institutions militaires, des soldats motivés et favorables au changement. L'évolution des forces n'est pas uniquement le résultat de pressions extérieures et du contrôle politique. Toutefois, pour que les réformateurs militaires puissent s'imposer, ils doivent être soutenus par le politique. Les décideurs civils ont pour obligation de trouver ces soldats et de lier des alliances avec eux pour diffuser la bonne parole du changement. On remarquera à quel point les conclusions de Rosen sont proches de certaines mesures mises en œuvre dans le contexte de la RMA. L'étude n'est pas restée lettre morte et a pu devenir une sorte de guide pratique, qui a ensuite connu une diffusion non négligeable dans les cercles concernés.

L'idée qu'il faut changer les forces armées américaines est ensuite relayée en dehors de l'institution par des think tanks. Les mêmes noms reviennent encore ici. En avril 2002, le Center for Strategic and Budgetary Assessment (CBSA) annonce par exemple qu'il a intégré Barry Watts dans son équipe de chercheurs ${ }^{35}$. A plusieurs égards, le CBSA ressemble à une "succursale» de l'office of Net Assessment. Il est dirigé par le Dr. Andrew F. Krepinevich Jr ${ }^{36}$, un ancien général du corps des Marines et un autre protégé de Marshall. Krepinevich, qui est donc passé par l'ONA, a rédigé des articles publiés dans le Joint Forces Quarterly, l'Armed Forces Journal, le National Interest, ou la Strategic Review. Il s'est également fait connaître en critiquant la façon dont la guerre du Vietnam avait été menée. Krepinevich avait aussi participé à la rédaction de 
Discriminate Deterrence sous la direction d'Albert Wohlstetter et de Fred Iklé ${ }^{37}$. Enfin, en 2002, il rend public un document d'analyse de la RMA à la demande de l'ONA ${ }^{38}$.

On notera qu'au sein d'un autre think tank, le Center for Security Policy, de Frank Gaffney Jr (un ancien reaganien), on semble aussi beaucoup apprécier Marshall. Par exemple, on s'y félicitera de ce que Marshall obtiendra davantage de responsabilités au sein du Pentagone lorsque Rumsfeld en prendra la tête ${ }^{39}$. Notons le slogan, on ne peut plus "réaliste», de ce centre: «la sécurité par la force». Ici aussi, le concept de changement des forces armées trouve un écho favorable.

Comme l'indique le titre de notre partie, les discours sur la RMA sont véritablement tentaculaires. Certes, on peut leur trouver un épicentre plus ou moins mythique à partir de l'ONA, mais ils connaissent ensuite une diffusion de plus en plus large. On notera que, parmi les individus et les centres de recherches mentionnés qui jouent un rôle de courroie de transmission, les accointances conservatrices et néoconservatrices s'affichent sans fausse pudeur. Une telle position politique ne peut certes être attribuée à toutes les personnes citées (entre autres par manque d'informations à leur égard). Par ailleurs, il est toujours possible que certains se soient joints au mouvement néoconservateur non pas sur une base politico-idéologique stricte, mais par accord avec quelques points précis de leur programme dans le contexte des questions de sécurité ${ }^{40}$. Comme Bruno Latour le mentionne, le nombre de personnes impliquées dans un projet technique fluctue sans cesse ${ }^{41}$. Il est difficile d'établir à l'avance une «liste» fiable de ceux qui seront intégrés dans le développement. Des alliés de circonstances peuvent apparaître en chemin et, a contrario, des acteurs de la première heure peuvent aussi s'éclipser. Cela semble bien être le cas pour la RMA.

Toutefois, on ne peut non plus passer sous silence le fait que la RMA se constitue progressivement en un discours hégémonique technique et use du capital symbolique de ceux qui la font connaître. Elle finit par se doter d'une légitimité de plus en plus forte ${ }^{42}$. Ceci est bien illustré par la parution du très populaire War and Anti-War, en 1993, par les futurologues Alvin et Heidi Toffler ${ }^{43}$. Le livre a joué un rôle important dans la prise de conscience que "quelque chose» de radical s'était passé dans le domaine des moyens techniques à disposition des forces armées. La RMA devenait un discours dans «l'air du temps ».

Discours hégémonique et action sur les identités

Le discours sur la RMA devenant hégémonique (discours qui recherche les alliés de circonstance et vise la diffusion la plus large possible), il convient de se poser la question de savoir comment les autres acteurs stratégiques vont se positionner vis-àvis de lui. Plus spécifiquement encore, il faut noter que l'existence d'un discours sur la RMA n'est pas simplement "confronté à d'autres acteurs ", il doit aussi être envisagé comme créateur potentiel de nouveaux acteurs. Certes, dans le cas qui nous intéresse, d'autres acteurs préexistent certainement au discours sur la RMA: il s'agit des différentes armes des forces armées américaines (en particulier l'US Air Force, l'US Navy et l'US Army). Traditionnellement, le domaine de la doctrine d'emploi - c'est-àdire les manuels opérationnels, tactiques et techniques - est de la responsabilité de chaque arme et non pas d'une instance centrale logée au Pentagone. La doctrine est par ailleurs considérée comme l'un des fondements essentiels de l'identité de chaque arme ; elle contribue à stabiliser l'image de chacune des armes quant à ce qu'elles sont et ce pour quoi elles existent. Elle est la transposition de la «raison d'être» de ces organisations et finit rapidement par devenir une culture stratégique ${ }^{44}$. C'est aussi de 
là que découle un intérêt institutionnel et bureaucratique. Il faut considérer qu'à travers la notion de culture stratégique, les différentes armes réalisent leur propre narration sociologique (ou « sociologisante ») des événements passés. C'est par ce biais que les acteurs et la conscience qu'ils ont d'eux-mêmes se créent et gagnent en cohésion.

Pendant les années 1990, ces identités ont été confrontées au discours de la RMA qui a d'abord été perçu comme un moyen de « faire plus avec moins de moyens ». La fin de la Guerre froide a ouvert la voie à des réductions de budget annoncées par une série de documents officiels : Base Force sous Bush, Bottom-Up Review - ou BUR - et Quadrenial Defense Review - QDR - sous Clinton. Les militaires ont perçu dans ce sillage une volonté politique de favoriser la coopération entre les différentes armes (concept de « jointness »), ce qui aurait pu conduire à des remises en cause des " chasses gardées " institutionnelles. Il devenait évident que, pour les tenants de la RMA, les réductions envisagées devaient s'accompagner d'un investissement plus important en moyens techniques. Les militaires ne se sont pas complètement alignés, cependant. Ils ont répondu à ces initiatives du pouvoir politique en tentant de maintenir leur capacité à court terme plutôt que d'investir à long terme. L'objectif des armes reste de tout faire pour être indispensable et maintenir, voire améliorer, leur statut, en particulier d'un point de vue budgétaire. Les documents BUR et QDR n'ont nullement incité à la transformation, mais ont plutôt ouvert la voie à une réduction de format des différentes armes selon des proportions identiques.

En d'autres termes, les discours sur la RMA vont permettre, et même obliger, les acteurs traditionnels à se dévoiler - ainsi que leurs identités et leurs intérêts - avec une certaine force. On peut considérer qu'il s'agit de prises de position «contrehégémoniques", remettant en cause le sens commun ou l'idéologie dominante. Le contexte est très "stratégique " car, historiquement, les bureaucraties ont l'habitude de défendre leur près-carré. Notons aussi que si les acteurs préexistent aux discours sur la RMA, ces derniers vont pourtant participer à la réaffirmation du statut d'acteur des différentes armes.

On constatera d'abord que l'articulation de discours "contre-hégémoniques » ne repose pas sur une réfutation de la RMA mais sur une tentative de redéfinition et de réappropriation, facilitée par le flou qui entoure encore un concept mal fixé. Il n'y a donc pas, de la part des militaires, de véritable "déréalisation", pour reprendre l'expression de Bruno Latour, du projet que constitue la RMA ${ }^{45}$. Il y aurait " déréalisation » si, par exemple, les militaires refusaient sciemment de donner réalité au projet, s'ils ne mettaient pas en pratique les concepts opérationnels qui en sont issus (pendant un conflit ou à l'entraînement); s'ils n'évoquent pas la RMA dans les académies pour officiers ou dans les manuels; s'ils ne mettent pas en œuvre les outils techniques et matériels de la RMA ; etc. Faire fonctionner la RMA nécessite de gagner l'adhésion d'individus, et un minimum d'adhésion est perceptible ${ }^{46}$. A partir de 1994, les militaires commencent à utiliser le concept. On le voit par exemple apparaitre dans une série d'études réalisées par le Strategic Studies Institute de l'Army War College ${ }^{47}$. Beaucoup d'officiers s'accordent à penser que la RMA prend place dans un environnement en mutation beaucoup plus large, d'un point de vue technique, social, politique, etc. L'ambiguïté du monde de l'après Guerre froide joue un rôle indéniable pas toujours très précis - dans cette lecture. Ce point mériterait également une analyse plus poussée. 

feront remarquer que les conceptions du changement au Pentagone durant ces années restent limitées et très graduelles ${ }^{49}$. Tout le monde parle le langage de la RMA, mais peu de changements réellement révolutionnaires s'enracinent. Pire encore, de nombreux projets militaires de la Guerre froide se poursuivent alors qu'ils avaient été initiés pour lutter contre le pacte de Varsovie. Parfois, seul le nom de ces mêmes projets change pour que les budgets continuent à affluer. Par ailleurs, on constate qu'un pacte tacite de non-agression se serait fait jour au sein des différentes armes. L'équilibre des forces bureaucratiques entre celles-ci est depuis bien longtemps une préoccupation du pouvoir politique civil. Cet équilibre est souvent vu comme synonyme d'inertie. Il empêcherait en particulier la réalisation de synergies et la mise en œuvre d'une meilleure coopération. Or, les civils à la tête de l'institution militaire ont toujours espéré que la coopération ferait prendre conscience des redondances de certaines fonctions assurées par plusieurs armes à la fois. En dépit d'une législation spéciale en la matière (l'amendement Goldwater-Nichols de 1986), la coopération entre les armes s'avère toujours problématique (en réalité, pour nombre de commentateurs, elle n'a jamais été satisfaisante depuis la fin de la Seconde Guerre mondiale) ${ }^{50}$. Dans le contexte de la RMA, on remarque qu'il existe pourtant une sorte de coopération mais que celle-ci vise à préserver les intérêts de chacun, au détriment de la volonté des politiques. Les documents interarmes Joint Vision 2010 et 2020 ont été pointés du doigt à ce propos car ils ne feraient que consacrer l'équilibre des forces institutionnelles.

\section{bureaucratique qu'est devenue la RMA depuis l'arrivée de Donald Rumsfeld au} Pentagone. Cette lutte s'est, depuis lors, matérialisée par une série de conflits de personnes et de décisions tranchantes.

Enfin, si un projet technique implique un nombre d'acteurs qui peut fortement évoluer avec le temps, la RMA n'a guère trouvé d'alliés ou d'opposants au sein des populations civiles. Il est possible d'opposer cet état de fait à ce qui se produit avec les questions techniques nucléaires qui ont parfois réussi à mobiliser un grand nombre d'organisations civiles ${ }^{51}$. Les prises de position en dehors de l'univers de la défense ne sont donc pas nombreuses, ou n'accèdent pas au champ social. L'intérêt pour la RMA est bien plus limité aujourd'hui que celui pour les armes de destruction massive. La RMA n'a donc pas été transformée en problème de société ${ }^{52}$.

Quelques mots sur la RMA au niveau international projets techniques recèlent une faisabilité et une réalité évolutives, dépendantes des interactions entre les acteurs ${ }^{53}$. Ces interactions jouent un rôle de premier plan dans les définitions et redéfinitions successives de ce que deviendra (ou, éventuellement, en cas d'échec, ce que ne deviendra pas) le projet. Par ces interactions aussi, les motivations des actions et les identités des acteurs peuvent changer (en plus du facteur temps qui a également un rôle dans ce processus). Par ailleurs, le projet peut acquérir en cours de route des dimensions transnationales.

Aujourd'hui, le concept de RMA est bien diffusé dans le langage militaire en dehors des Etats-Unis, en particulier au sein des Etats anglo-saxons. Non des moindres, les Etats 
considérés comme les plus proches alliés traditionnels des Etats-Unis, la GrandeBretagne et les Pays-Bas par exemple, paraissent très attentifs aux évolutions en cours. Dans un second temps, les autres Etats de l'OTAN - mais pas uniquement - accordent également une attention au processus de transformation. L'objectif de l'article n'est cependant pas de suivre pas à pas l'extension du discours sur la RMA en dehors des Etats-Unis, les réflexions qui suivent restent principalement des pistes. Cependant on pourrait certainement y relever l'émergence d'un discours transnational contraignant les forces armées à travers le monde ${ }^{54}$. On pourrait probablement aussi y trouver, dans quelques cas, des éléments relatifs à l'affirmation symbolique de la souveraineté étatique que l'on décèle traditionnellement dans la possession de certains équipements militaires ${ }^{55}$. On devrait aussi se demander jusqu'à quel point la diffusion du discours sur la RMA ne repose pas aussi sur la constitution d'une "élite cosmopolite" d'officiers, d'industriels et d'intellectuels de la défense socialisée à une même série d'idées stratégico-techniques, directement influencées par l'hégémonie du discours américain et sa facilité de circulation ${ }^{56}$. Enfin, trop souvent, les considérations sur la diffusion de la RMA ne s'embarrassent guère de penser, plus globalement, aux conséquences éventuelles en dehors de l'univers militaire. Les historiens sont pourtant là pour rappeler que l'adoption de techniques militaires a souvent eu un impact beaucoup plus large - parfois désastreux - dans les sociétés concernées. Plus encore, se trouve posée la question de l'indépendance relative des forces armées qui acceptent les effets d'une transformation technique ${ }^{57}$. Au total, il conviendrait de décrypter de façon aussi systématique que possible la diffusion hors Etats-Unis des discours sur la RMA et de les replacer dans leurs contextes institutionnels, sociaux, économiques et stratégiques. En un mot, il faudrait se demander quels sont les canaux d'extension du discours sur la RMA.

Toutefois, dans la prise en compte de la diffusion du discours sur la RMA, on ne perdra pas de vue, en suivant Bruno Latour, que la signification du concept peut évoluer ${ }^{58}$. En se diffusant, le concept peut gagner en indépendance par rapport à ceux qui l'ont développé à l'origine. En d'autres termes, le concept de RMA peut se retrouver plus ou moins fortement redéfini dans ce contexte. C'est une question qu'il faudrait se poser en particulier concernant les discours sur la RMA au sein de l'OTAN et des pays européens actuellement ${ }^{59}$.

L'absence de définition ferme de la RMA autorise de nombreuses négociations, tractations, oppositions entre les acteurs impliqués (ou les acteurs qui s'impliquent). La réalité et la faisabilité du projet deviennent des concepts progressifs découlant des résultats des interactions. Par ce biais surgit le concept que Latour nomme le "blackboxing ", en référence à la boîte noire qui occulte les nombreuses dynamiques sociales à l'œuvre au profit d'un schéma généralisateur, souvent sous forme de narrations qui rationalisent après-coup ${ }^{60}$. La boîte noire n'est pourtant pas totalement opaque. Les processus de négociation laissent des traces. Mais l'une des caractéristiques du blackboxing est de faire sortir un projet qui ne correspond plus aux critères de départ. Le projet pensé initialement n'est pas le projet réalisé en fin de course. La RMA deviendra - ou ne deviendra pas - ce que les acteurs en font. De même, les critères qui autorisent à affirmer la réussite ou l'échec du projet peuvent aussi s'avérer évolutifs. Ces critères relèvent souvent d'abord d'une perception linéaire et généralisatrice d'un projet. Ajoutons néanmoins que dans le cas où les tentatives de définition et de 
redéfinition deviennent pléthoriques, il existe un risque de dénaturation qui peut tout simplement faire échouer le projet.

Enfin, les acteurs contextualisent le projet. Ce dernier n'est nullement doté d'emblée d'un contexte indiscutable. Il s'en donne un grâce aux acteurs et à leurs tractations, d'où les tentatives de redéfinir la culture militaire américaine aujourd'hui. C'est aussi à travers la constitution du contexte que les acteurs se mettent d'accord sur la façon dont il faut interpréter le monde et agir en conséquence de cette création sociale (par exemple, le fait de considérer que le contexte de la RMA doit être cherché dans l'existence d'un monde anarchique où les ennemis potentiels sont nombreux ${ }^{61}$ ).

Plus encore, cela signifie que le projet de la RMA entretient des liens essentiels avec des présupposés idéels et/ou idéologiques. Ce sont ces derniers que l'on a tenté de mettre en évidence pour déconstruire le concept. Ils nous ont permis de présenter les rapports de forces, les alliances et l'affirmation des identités qui traversent les discours sur la RMA. Au final, il faut insister sur le fait qu'en aucun cas la RMA ne peut être étudiée uniquement sous un jour technique. Le projet est ancré dans une série de représentations relatives aux identités des acteurs militaires, à la nature des relations internationales, à la place des Etats-Unis dans le monde, à l'utilité de la technique, etc. Par cette entremise, nous espérons aussi mettre en garde contre l'adoption trop rapide de moyens techniques militaires apparus dans le sillage de la RMA en l'absence d'une solide réflexion préalable.

\section{NOTES}

1.. « RMA » ou « RAM » pour « Révolution dans les affaires militaires ».

2.. Définition officielle absente de la Joint Publication 1-02, Department of Defense Dictionary of Military and Associated Terms, 12 avril 2001 (revue le 9 janvier 2003).

3.. Nous désirons adresser nos remerciements au comité de lecture de la revue Cultures \& Conflits pour ses commentaires.

4.. « Aramis » est le nom donné à un projet français de transport en commun plus autonome, une sorte de métro amélioré. Ce projet technique fut un échec, ce que rapporte Bruno Latour dans Aramis ou l'amour des techniques, Paris, La Découverte, 1993. 5.. Voir aussi Weldes J., " Bureaucratic politics: a critical constructivist assessment ", Mershon International Studies Review, vol. 42, n², novembre 1998, pp. 216-225.

6.. Sur Marshall, on lira : Silverstein K., «Buck Rogers rides again », The Nation, 25 octobre 1999 (consulté sur www.comw.org/rma/fulltext/buck.html) ; Vest J., « The new Marshall plan », InTheseTimes.com, 2 avril 2001 (consulté sur www.inthesetimes.com/ issue/25/09/vest2509.htm) ; Lemann N., " Bush watch, Bush's RMA », 16 juillet 2001 (www.bushwatch.com/rma.htm) ; McGray D., « The Marshall plan », Wired, 11/2, février 2003 (www.wired.com/wiredarchives/11.02/marshall.html).

7.. Kaplan F., The Wizards of Armageddon, New York, Touchstone, 1983.

8.. Sionneau B., " Réseaux conservateurs et nouvelle doctrine américaine de sécurité ", in Annuaire français de relations internationales, IV, Bruxelles, Bruylant, 2003, pp. 498-531. 
9.. Der Derian J., " The illusion of a grand strategy », The New York Times, 25 mai 2001. 10.. Sur le prophétisme de la guerre, on lira Clarke I.F., Voices Prophesying War. Future Wars 1763-3749, Oxford/New York, Oxford University Press, 1992.

11.. Vest J., " The dubious genius of Andrew Marshall », The American Prospect, 10 novembre 1997 (www.prospect.org/webfeatures/2001/02/vest-j-02-15.html).

12.. Latour B., Aramis, op. cit., pp. 136-137.

13. Latour B., La Science en action. Introduction à la sociologie des sciences, Paris, La Découverte, 2005, p. 64.

14.. Geertz C., The Interpretation of Culture, New York, Basic Books, 1973, p. 201 et suivantes.

15.. Notre traduction. McKitrick J., Blackwell J., Littlepage F., Kraus G., Blanchfield R., Hill D., « The Revolution in military affairs », in Schneider B.R., Grinter L.E., (dir.), Battlefield of the Future. 21st Century Warfare Issues, Air War College, septembre 1995 (www.airpower.maxwell.af.mil/airchronicles/battle/front.html). On remarquera que le texte cite Marshall sans références précises.

16. Pour une synthèse des ces théories, voir notre article « Théorie des relations internationales, doctrines militaires et pensée stratégique et de sécurité ", Les Champs de Mars, 2nd semestre 2003, pp. 145-172.

17.. Sur les ramifications d'un projet technique, dans Aramis, Latour s'inspire du rhizome de Deleuze G. et Guattari F., Milles Plateaux. Capitalisme et schizophrénie 2, Paris, Minuit, 1980, pp. 9-37.

18.. Kaplan F., The Wizards of Armageddon, op. cit.

19.. Rosecrance R., « Albert Wohlstetter », in Baylis J., Garnett J. (dir.), Makers of Nuclear Strategy, Londres, Pinter, 1991, pp. 57-69.

20.. L'une de ses dernières actions notables dans le domaine est sa participation à : Iklé F.C., Wohlstetter A., "Discriminate Deterrence”, un rapport sur la nouvelle stratégie américaine, (trad. de l'anglais), GRIP « notes et documents », n¹29-130, janvier-février 1989.

21.. Qui s'exprime au sein du PNAC ou Project for a New American Century (www.newamericancentury.org/).

22.. Keaney T.A., Cohen E.A., Gulf War Air Power Survey Summary Report, Washington D.C., GPO, 1993.

23.. Krepinevich y donne par exemple un enseignement intitulé Net Assessment. Pour plus d'informations sur la SAIS et ses membres, voir : www.sais-jhu.edu/programs/ir/ strategic/faculty.

24.. « Northrop Grumman's analysis center: thinking through the issues », 2000 (voir www.northgrum.com). Grumman est une société régulièrement impliquée dans les débats sur les modernisations des forces armées.

25.. United States Department of Defense, « New director of program analysis and evaluation named », News Release, $\mathrm{n}^{\circ} 188-01$, 1er mai 2001 ; « Director, program analysis and evaluation, Barry D. Watts », DDN Biographies, (www.defensedaily.com/biographies/ osd/dirproanalysis_bio.htm).

26. Roche et Watts sont aussi les auteurs de "Choosing analytic measures », The Journal of Strategic Studies, vol. 14, n² , juin 1991, pp. 165-209.

27.. " President Bush to nominate four individuals to serve in his administration ", The White House, Office of Press Secretary, 24 avril 2001 (www.whitehouse.gov/news/releases/ 2001/04/20010424-2.html). 
28.. Murray W., Millet A.R., Military Innovation in the Interwar Period, Cambridge, Cambridge University Press, 1996. Voir aussi : Sullivan B.R., « Are we really ready for an RMA? », Joint Forces Quarterly, été 1996, pp. 112-113.

29.. Marshall A.W., « The 1995 RMA essay contest: a postscript », Joint Forces Quarterly, hiver 1995-1996, p. 81.

30.. Murray W., « Thinking about Revolutions in military affairs ", Joint Forces Quarterly, été 1997, pp. 69-76.

31.. Murray W. "Innovation: past and future », Joint Forces Quarterly, été 1996, p. 60.

32.. Texte consulté le 25 octobre 2003 sur www.newamericancentury.org/ defnov1097.htm.

33.. Hussain K., " American dreams. Intellectual roots of neo-conservative thinking », consulté sur www.studien-von-zeitfragen.de/Weltmacht/American_Dreams/ american_dreams.html (initialement publié en mars 2003 dans le Herald Pakistan).

34.. Rosen S.P., Winning the Next War. Innovation and the Modern Military, Ithaca/Londres, Cornell University Press, 1991.

35.. Frye A., " CSBA hires Barry Watts as senior fellow », Press Release, 26 août 2002 (consulté sur www.csbaonline.org).

36.. On trouvera son curriculum vitae sur www.csbaonline.org.

37.. Iklé F.C., Wohlstetter A., « Discriminate deterrence », op. cit.

38.. Krepinevich A.F., The Military-Technical Revolution: A Preliminary Assessment, CSBA, 2002 (disponible sur www.csbaonline.org). L'avant-propos est signé par A. Marshall.

39.. Voir la note numérotée 01-D13 de février 2003 sur le site www.security-policy.org/. 40.. L'amiral Owens est peut-être un bon exemple d'un défenseur de la RMA que l'on ne peut a priori pas taxer de néoconservatisme. Owens W.A., « Creating a Military revolution ", in Farrell T., Terriff T. (dir.), The Sources of Military Change: Culture, Politics, Technology, Londres/Boulder, Lynne Rienner, 2002, pp. 205-219.

41. Latour B., Aramis, op. cit. pp. 66-67.

42.. Voir Bigo D., « Les débats en relations internationales et leur lien avec le monde de la sécurité ", Cultures \& Conflits, automne-hiver 1995 (disponible sur www.conflits.org) ; Klein B.S., Strategic Studies and World Order. The Global Politics of Deterrence, Cambridge, Cambridge University Press, 1994.

43.. Toffler A. et H., Guerre et contre-guerre. Survivre à l'aube du xxie siècle, (trad. de l'anglais), Paris, Fayard, 1994.

44.. La culture stratégique étant conçue comme une série de préférences stratégiques qui agissent sur le long terme et sont dotées d'une grande légitimité sur le plan des prescriptions. Johnston A.I., Cultural Realism: Strategic Culture and Grand Strategy in Chinese History, Princeton, Princeton University Press, 1995.

45.. Latour B., Aramis, op. cit., p. 78.

46.. On pourrait cependant se demander si les actions violentes asymétriques d'un adversaire ne sont pas une sorte de déréalisation.

47. Disponibles en ligne sur www.carlisle.army.mil/ssi.

48. Parmi ceux-ci, mentionnons par exemple le Field Manual 100-5, Operations de 1993, Force XXI, le TRADOC Pamphlet 525-5, les livres blancs Global Reach, Global Power et Global Presence de l'US Air Force, Joint Vision 2010, Forward... From the Sea, Army Vision 2010, Report on the Army After Next, Global Engagement, etc. Mentionnons aussi l'implication d'une multitude de centres de recherches militaires parmi lesquels le Joint Requirements Oversight Council, la National Defense University, l'Army War College, le Future Technologies Institute, les Battle Labs, l'Air University, l'Air Force Information Warfare Center, le Fleet 
Information Warfare Center, le Marine Corps Commandant's Warfighting Laboratory. Chairman of the Joint Chief of Staff, Joint Vision 2010, Washington, Office of the Chairman, s.d. (1996, suivi par un Joint Vision 2020 en 1997) ; US Navy, Forward... From the Sea, Washington, Department of the Navy, 1994 ; US Army, Army Vision 2010, Washington, Department of the Army, [s.d.] (1996) ; US Army, Knowledge and Speed: The Annual Report on the Army After Next Project to the Chief of Staff of the Army, Fort Monroe, US Army TRADOC, 1997 ; US Air Force, Global Engagement: A Vision for the US Air Force in the 21st Century, Washington, Department of the Air Force, 1997. Voir aussi : Durand E., Les Transformations de l'US Army, Les Etudes de l'IFRI, n¹, juillet 2003.

49.. Krepinevich A., « Why no transformation?», The National Interest, 4 février 1999 (consulté sur www.csbaonline.org).

50.. Locher J.R., « Has it worked? The Goldwater-Nichols rorganization Act », Naval War College Review, automne 2001 (www.nwc.navy.mil/press/Review/2001/Autumn/art5au1.htm).

51.. Boyer P., « From ativism to apathy: the American people and nuclear weapons, 1963-1980 ", The Journal of American History, vol. 70, n² 4, mars 1984, pp. 821-844.

52. Voir malgré tout le Project on Defense Alternatives (www.comw.org/pda/) et le Center for Defense Information (www.cdi.org).

53.. Latour B., Aramis, op. cit., pp. 50-75.

54.. Léonard S., Balzacq T., "L'impact de la révolution dans les affaires militaires sur la “culture stratégique" de l'OTAN ", in Balzacq T. et de Nève A. (dir.), La Révolution dans les affaires militaires, (préf. par Bruno Colson), Paris, Economica/ISC, 2003, pp. 157-185.

55.. Par exemple : Kinsella D., Jugdep S., « Symbols of statehood: military industrialization and public discourse in India ", Review of International Studies, vol. 27, 2002, pp. 353-373.

56. Sur cette thèse, voir Dezalay Y., « Les courtiers de l'international : Héritiers cosmopolites, mercenaires de l'impérialisme et missionnaires de l'universel ", Actes de la recherche en sciences sociales, $\mathrm{n}^{\circ} 151-152$, mars 2004, pp. 5-35.

57.. Ces questions sont illustrées dans Ralston D.B., Importing the European Army. The Introduction of European Military Techniques and Institutions into the Extra-European World, Chicago/Londres, University of Chicago Press, 1990.

58.. Latour B., Aramis, op. cit., p. 180.

59.. Voir de Neve A., Mathieu R., Les Armées d'Europe face aux défis capacitaires et technologiques, Bruxelles, Bruylant/LGDJ, 2005.

60.. Dans l'univers de la défense, voir également McKenzie D., Spinardi G. dans leurs deux articles « The shaping of nuclear weapons system technology: US fleet ballistic missile guidance and navigation I: from Polaris to Poseidon ", Social Studies of Science, vol. 18, n³, août 1988, pp. 419-463; « The shaping of nuclear weapons system technology: US fleet ballistic missile guidance and navigation II: "Going for Broke" The path for trident II », Social Studies of Science, vol. 18, n², novembre 1988, pp. 581-624.

61.. Wendt A., « Anarchy is what states make of it: the social construction of power politics », International Organization, vol. 46, n² 2, été 1992, pp. 391-425. 


\section{RÉSUMÉS}

L'objectif de l'analyse consiste à appliquer la méthode développée par Bruno Latour - pour étudier le projet technique "Aramis" - à l'élaboration d'une Révolution dans les affaires militaires (RAM) aux Etats-Unis. Par cette méthode, il est possible de décrypter les discours constitutifs d'un projet militaire, de mettre à jour les identités des acteurs concernés ainsi que leurs relations. En fin de compte, l'aspect construit de la RMA est mis en évidence.

The aim of the analysis is to apply the method developed by Bruno Latour - in order to study the technical project Aramis - to the creation of a Revolution in Military Affairs (RMA) in the United States. Thanks to this method, it is possible to decypher the constituting discourses of a military project, to highlight the identities of the actors involved and their relations. Finally, the emphasis is put on the constructed nature of the RMA.

\section{INDEX}

Mots-clés : Doctrine militaire, guerre, Militaires, Stratégie

Keywords : RMA

Thèmes : Pentagone (US Department of Defence)

\section{AUTEUR}

\section{CHRISTOPHE WASINSKI}

Christophe Wasinski est assistant à l'université libre de Bruxelles au sein du REPI (Réseau d'étude en politique internationale) et aux facultés universitaires Notre-Dame de la Paix de Namur. Il est également membre du Réseau multidisciplinaire en études stratégiques (www.rmes.be). Il a récemment contribué à l'ouvrage : Delcourt B., Duez D., Remacle E., La Guerre d'Irak : prélude d'un nouvel ordre international ? (Bruxelles, PIE-Peter Lang, 2004). 